Virginia Commonwealth University

VCU Scholars Compass

2005

\title{
First-principles study of ferromagnetic coupling in Zn1-xCrxTe thin film
}

Q.Wang

Virginia Commonwealth University

Q.Sun

Virginia Commonwealth University

P. Jena

Virginia Commonwealth University, pjena@vcu.edu

Y. Kawazoe

Tohoku University

Follow this and additional works at: http://scholarscompass.vcu.edu/phys_pubs

Part of the Physics Commons

Wang, Q. Sun, Q. Jena, P., et al. First-principles study of ferromagnetic coupling in Zn1-xCrxTe thin film. Journal of Applied Physics 97, 043904 (2005). Copyright (C 2005 AIP Publishing LLC.

\section{Downloaded from}

http://scholarscompass.vcu.edu/phys_pubs/127

This Article is brought to you for free and open access by the Dept. of Physics at VCU Scholars Compass. It has been accepted for inclusion in Physics Publications by an authorized administrator of VCU Scholars Compass. For more information, please contact libcompass@vcu.edu. 


\title{
First-principles study of ferromagnetic coupling in $\mathrm{Zn}_{1-x} \mathrm{Cr}_{x} \mathrm{Te}$ thin film
}

\author{
Q. Wang, Q. Sun, and P. Jena ${ }^{a)}$ \\ Physics Department, Virginia Commonwealth University, Richmond, Virginia 23284 \\ Y. Kawazoe \\ Institute for Material Research, Tohoku University, Sendai, 980-8577, Japan
}

(Received 1 September 2004; accepted 2 December 2004; published online 25 January 2005)

\begin{abstract}
Using gradient-corrected density functional theory and supercell technique, we have calculated total energies, electronic structure, and magnetic properties of $\mathrm{Cr}$-doped $\mathrm{ZnTe}$ in both bulk and thin-film configurations. Calculations with full geometry optimization for a $\mathrm{Zn}_{1-x} \mathrm{Cr}_{x} \mathrm{Te}$ supercell were carried out for different $\mathrm{Cr}$ concentrations $(x=0.095,0.143$, and 0.19) and by varying the sites $\mathrm{Cr}$ atoms occupy. We show that the ferromagnetic phase of $\mathrm{Zn}_{1-x} \mathrm{Cr}_{x} \mathrm{Te}$ in both bulk and thin film is energetically the most preferable state irrespective of the concentration and/or site occupation of the $\mathrm{Cr}$ atom. The strong hybridization between $\mathrm{Cr} 3 d$ and $\mathrm{Te} 5 p$ states is found to be responsible for the ferromagnetic coupling, in agreement with recent experiments. (C) 2005 American Institute of Physics. [DOI: 10.1063/1.1851013]
\end{abstract}

\section{INTRODUCTION}

The discovery of ferromagnetism in GaMnAs (Ref. 1) and InMnAs (Ref. 2) has given rise to a great deal of interest in the last decade in the study of dilute magnetic semiconductor (DMS) systems. Unfortunately, the highest Curie temperature achieved in the $(\mathrm{Ga}, \mathrm{Mn})$ As system, even with heavy doping, is $110 \mathrm{~K}$. This is well below room temperature and these DMS systems are not suitable for practical applications. Therefore, great effort has been devoted to the synthesis and characterization of types of DMS materials by using various experimental techniques. Although roomtemperature ferromagnetism has been reported in many systems, such as $\mathrm{GaN}: \mathrm{Mn},{ }^{3-13} \mathrm{GaN}: \mathrm{Cr},{ }^{14,15} \mathrm{TiO}_{2}: \mathrm{Co},{ }^{16}$ $\mathrm{ZnO}: \mathrm{Co},{ }^{17} \mathrm{CdGeP}_{2}: \mathrm{Mn},{ }^{18}$ and $\mathrm{ZnO}: \mathrm{Mn},{ }^{19,20}$ there is considerable controversy regarding the origin of ferromagnetism in these systems. For example, is ferromagnetism caused by $s p-d$ exchange interaction, which is characteristic of DMS systems, or is it due to magnetic precipitates in the sample?

Recently magnetic circular dichroism (MCD) measurements ${ }^{21}$ were used to clarify the origin of ferromagnetism in DMS systems. Three DMS systems that exhibit ferromagnetic order unambiguously are $\mathrm{GaMnAs},{ }^{22}$ InMnAs, ${ }^{23}$ and $\mathrm{ZnCrTe} .{ }^{24-26}$ The former two are the prototype DMS in III-V family and have been studied extensively. The last one is the first II-VI DMS where high- $T_{c}$ ferromagnetism is reliably detected. Saito $e t a l .{ }^{24}$ have succeeded in synthesizing a $\mathrm{Zn}_{1-x} \mathrm{Cr}_{x} \mathrm{Te}$ thin film using molecular-beam epitaxy method that under heavy doping $(20 \% \mathrm{Cr})$ exhibited room-temperature ferromagnetism. While ferromagnetism in bulk $\mathrm{ZnCrTe}$ system has been studied theoretically, ${ }^{27-32}$ no calculations are available to our knowledge on the thin film or surface. In this paper, we present a theoretical study using density functional theory and the slab supercell model.

\footnotetext{
a) Author to whom correspondence should be addressed; electronic mail: pjena@vcu.edu
}

\section{THEORETICAL APPROACH}

We have modeled the ZnTe thin film by a seven-layer slab having zinc-blende structure along (110) direction (see Fig. 1), which contains 84 atoms (42 $\mathrm{Zn}$ and $42 \mathrm{Te}$ atoms). To preserve symmetry, the top and bottom layers of the slab were taken to be identical, and each slab was separated from the other by a vacuum region of $10 \AA$. The central three layers were fixed at their bulk configuration, while the two surface layers on either side of the slab were allowed to relax without any symmetry constraint. To study the magnetic coupling between $\mathrm{Cr}$ atoms, we considered three different con-

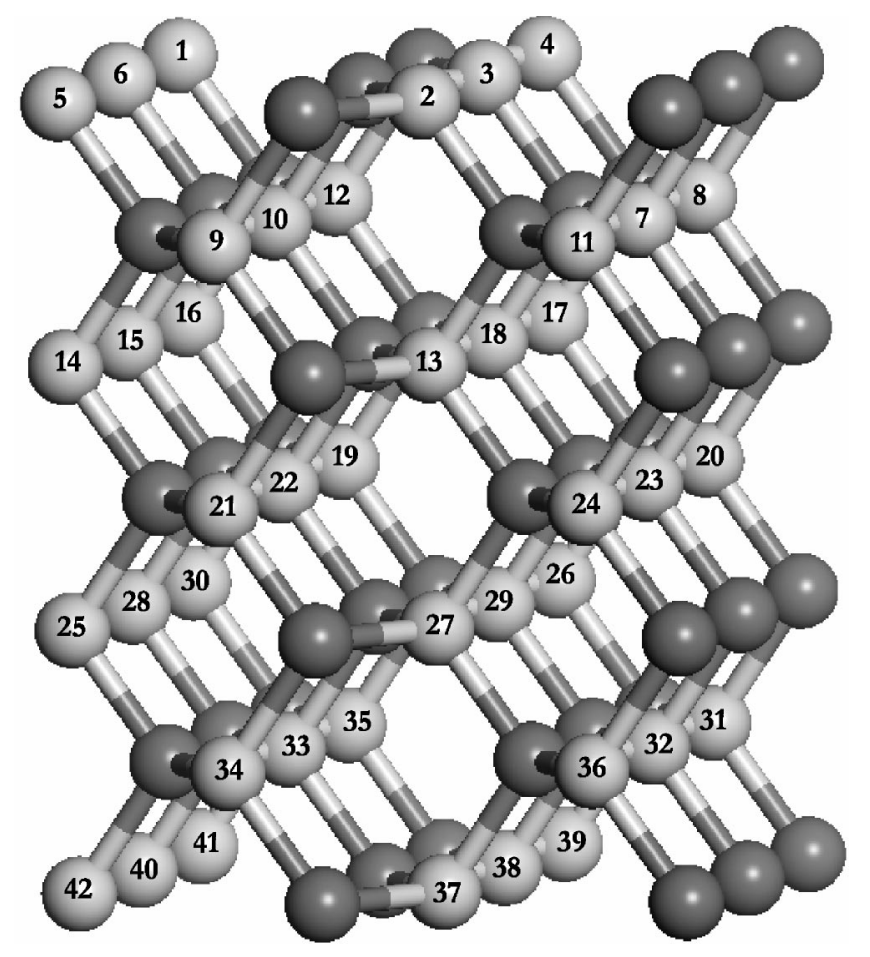

FIG. 1. The schematic representation of ZnTe (110) zinc-blende slab supercell consisting of $42 \mathrm{Zn}$ and $42 \mathrm{Te}$ atoms. The lighter and numbered balls are $\mathrm{Zn}$, the darker balls are Te. 


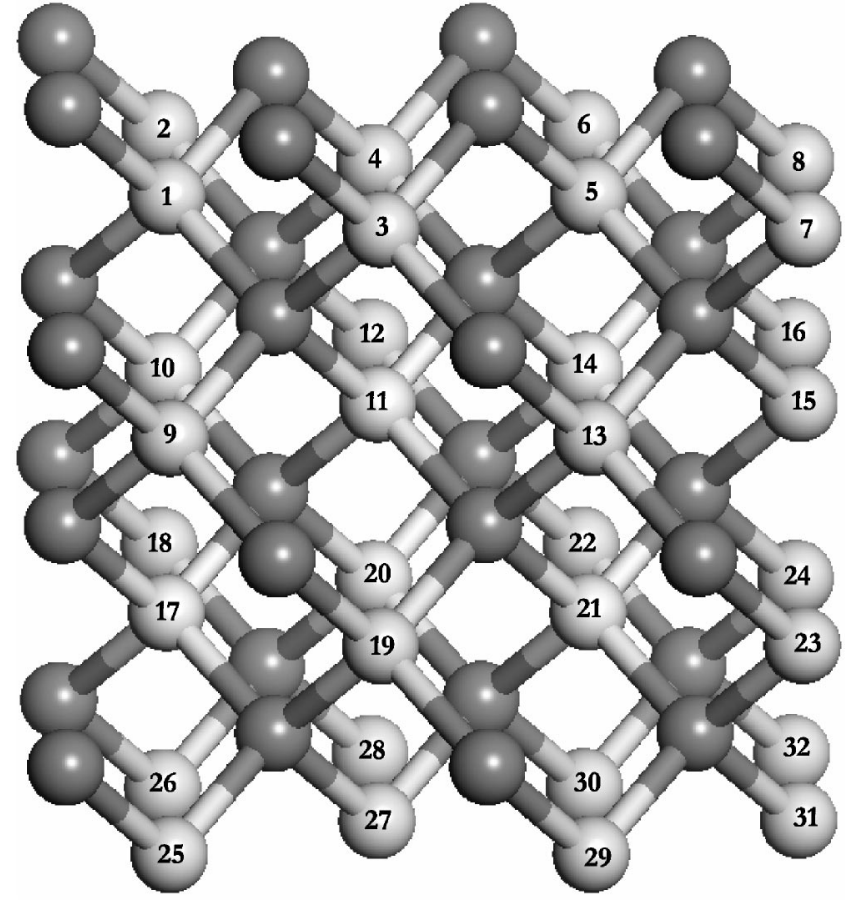

FIG. 2. The schematic representation of $(2 \times 2 \times 2)$ bulk zinc blende $\mathrm{ZnTe}$ supercell consisting of $32 \mathrm{Zn}$ and $32 \mathrm{Te}$ atoms. The lighter and numbered balls are $\mathrm{Zn}$, the darker balls are Te.

centrations: $9.5 \%, 14.3 \%$, and $19.0 \%$. Calculations of total energies, forces, and optimization of geometries were carried out using a plane-wave basis set with the projector augmented plane wave method $^{33}$ as implemented by VASP software. $^{34} K$-point convergence was achieved with $(4 \times 4$ $\times 1)$ Monkhorst-Pack ${ }^{35}$ grid, and tests with up to $(6 \times 6$ $\times 2$ ) Monkhorst $k$-point mesh were made to ensure the accuracy of our calculation. The energy cutoff was set at $300 \mathrm{eV}$ and the convergence in energy and force were $10^{-4} \mathrm{eV}$ and $3 \times 10^{-3} \mathrm{eV} / \AA$, respectively. Using the same technique, we also performed calculations for bulk $\mathrm{Zn}_{0.81} \mathrm{Cr}_{0.19}$ Te by considering a $(2 \times 2 \times 2)$ zinc-blende 64-atom $\mathrm{ZnTe}$ supercell (as shown in Fig. 2) and using $32 k$-points in the irreducible Brillouin zone.

\section{RESULTS}

\section{A. Bulk (Zn, Cr)Te}

Although the ferromagnetism in bulk Cr-doped ZnTe has been theoretically studied, ${ }^{27-32}$ the atomic positions were either not relaxed or only partially optimized. We first present our results on bulk Cr-doped ZnTe and compare them with earlier calculations. We choose the doping concentration to be $19 \%$, which is close to the experimental concentration. $^{24-26}$ This concentration corresponds to replacing six $\mathrm{Zn}$ atoms with $\mathrm{Cr}$ in the $\mathrm{Zn}_{32} \mathrm{Te}_{32}$ supercell. There are many possible positions for $\mathrm{Cr}$ atoms to reside. We have studied four different configurations in which $\mathrm{Cr}$ atoms are allowed to either cluster in different forms or to remain isolated. First, we replaced six nearest-neighbor $\mathrm{Zn}$ atoms with $\mathrm{Cr}$ in the sites marked Nos. 10, 12, 14, 21, 19, and 17 in Fig. 2 (configuration I). Configuration II corresponds to replacing two groups of Zn sites marked Nos. $(3,13,19)$ and $(4,14,20)$, where each group consists of three nearest-neighbor atoms. Configuration III corresponds to the replacement of sites Nos. $(3,5),(10,12)$, and $(19,21)$, which formed three groups of two nearest-neighbor $\mathrm{Zn}$ sites. Configuration IV relates to the substitution of six $\mathrm{Zn}$ sites where $\mathrm{Cr}$ atoms do not occupy nearest-neighbor sites. These are marked Nos. 7 , $3,10,14,19$, and 23. This corresponds to a configuration where $\mathrm{Cr}$ atoms are isolated and do not interact directly with each other. We found that $\mathrm{Cr}$ atoms prefer to cluster around Te atoms, and the ferromagnetic (FM) state of configuration I is the most stable one among all the four configurations studied. The calculation of antiferromagnetic (AFM) state in configuration I was done by assigning an initial moment of $5 \mu_{\mathrm{B}}$ to $\mathrm{Cr}$ atoms at sites 10,14 , and 19 , and $-5 \mu_{\mathrm{B}}$ at sites 12,21 , and 17. After full optimization, it was found that the AFM state is $0.680 \mathrm{eV}$ higher in energy than the FM state, which is comparable to the values obtained with linear muffin tin orbital method for the $\mathrm{Cr}$ concentration of $12.5 \% .^{29}$ Similar calculations have been performed for configurations II, III, and IV. The FM states of configuration II, III, and IV are $0.489,0.492$ and $0.519 \mathrm{eV}$ higher in energy than the most stable state, respectively. The optimized lattice constant at this concentration (19\%) is $6.19 \AA$. This is in agreement with the predicted value of $6.14 \AA$, which is obtained using a linear relationship between the lattice constant $a_{L}$ and $\mathrm{Cr}$ concentration $x\left(a_{L}=0.163 x+6.104\right) .{ }^{26}$ Previous studies have reported bulk $\mathrm{ZnCrTe}$ to be $\mathrm{FM},{ }^{27-32}$ while we found here that the coupling between $\mathrm{Cr}$ spins remains FM before and after the structures were fully optimized. In the stable configuration, the magnetic moment of each $\mathrm{Cr}$ atom is $3.55 \mu_{\mathrm{B}}$, and arises mainly from $\mathrm{Cr} 3 d$ orbital $\left(3.48 \mu_{\mathrm{B}}\right)$ with a small contribution from $4 s\left(0.03 \mu_{\mathrm{B}}\right)$ and $5 p\left(0.03 \mu_{\mathrm{B}}\right)$ due to the $s p$ and $d$ hybridization of $\mathrm{Cr}$. It is found that all the Te atoms bridging the $\mathrm{Cr}$ atoms are antiferromagnetically polarized, similar to what was found with the full-potential linearized augmented plane wave method for the $\mathrm{Cr}$ concentrations of $25 \%$ and $50 \% .^{30,31}$ This AFM polarization for the carriers is consistent with the $p$ - $d$ exchange interaction mechanism required for ferromagnetism via carrier mediation. The total density of states (DOS) and partial DOS around the $\mathrm{Cr}$ atom are plotted in Figs. 3(a) and 3(b), respectively. Note that the Fermi energy passes through the spin-up DOS and through the gap in spin-down DOS [Fig. 3(a)]. The system, therefore, is half metallic. The DOS at the Fermi energy is dominated by the Cr $3 d$ states [Fig. 3(b)].

\section{B. $(\mathrm{Zn}, \mathrm{Cr}) \mathrm{Te}$ thin film}

In this section, we begin discussion on the atomic structure of undoped ZnTe thin film. The surface reconstruction calculation of this thin film was carried out by optimizing the geometry of the slab. The relaxation energy per surface dimer is found to be $0.24 \mathrm{eV}$. The bond length between $\mathrm{Zn}$ and Te atoms on the surface contracted by $-1.67 \%$, while that in the subsurface layer expanded by $1.1 \%$ as compared to the bulk. The DOS of undoped ZnTe thin film is shown in Fig. 4(a), which is characterized by a large band gap.

To study the site preference of a $\mathrm{Cr}$ atom, we have replaced a single $\mathrm{Zn}$ atom with $\mathrm{Cr}$ on both the surface layer 

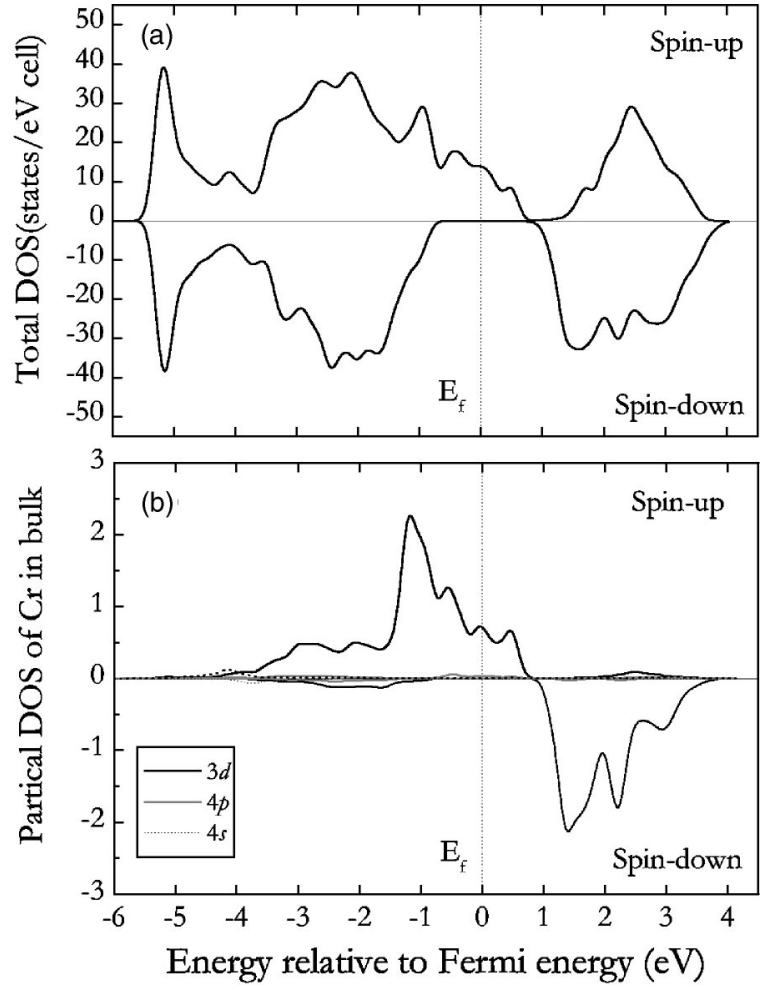

FIG. 3. (a) Total DOS of Cr-doped bulk ZnTe corresponding to 19\% doping. (b) Partial DOS of $\mathrm{Cr}$ at site No. 19 in Fig. 2.

(marked No. 3) and the subsurface layer (marked No. 10) in Fig. 1, separately. To preserve the symmetry, corresponding $\mathrm{Zn}$ atoms on the lower half of the slab (marked Nos. 38 and 33) were also replaced, respectively. Since the distance between these two Cr atoms (No. 3 and No. 38; No. 10 and No. 33) is quite large (12.07 $\AA$ and $8.91 \AA$, respectively), one can assume that they do not interact with each other. The corresponding supercell has the composition $\mathrm{Zn}_{40} \mathrm{Cr}_{2} \mathrm{Te}_{42}$. From the total energy calculations, we find that $\mathrm{Cr}$ atom prefers to reside on the subsurface site, which is $0.05 \mathrm{eV} / \mathrm{Cr}$ atom lower in energy than that of the surface site. This is in contrast to $(\mathrm{Ga}, \mathrm{Mn}) \mathrm{N}$ where $\mathrm{Mn}$ prefers the surface site. ${ }^{36} \mathrm{In}$ ( $\mathrm{Zn}, \mathrm{Mn}) \mathrm{O}$ system, on the other hand, Mn exhibited no site preference. ${ }^{37}$

To study the magnetic coupling between $\mathrm{Cr}$ atoms, it is necessary to replace two or more neighboring $\mathrm{Zn}$ sites in Fig. 1 with $\mathrm{Cr}$. We have studied three different concentrations of $\mathrm{Cr}$, and the results are given in the following.

\section{1. $\mathrm{Zn}_{0.905} \mathrm{Cr}_{0.095} \mathrm{Te}$}

This composition corresponds to having four $\mathrm{Zn}$ atoms replaced by $\mathrm{Cr}$ in the supercell $\left(\mathrm{Zn}_{42} \mathrm{Te}_{42}\right.$ in Fig. 1) forming $\mathrm{Zn}_{38} \mathrm{Cr}_{4} \mathrm{Te}_{42}$. Since it is a priori not clear which $\mathrm{Zn}$ sites in the supercell will be preferred by $\mathrm{Cr}$, we studied five different configurations. For each configuration, we replaced two $\mathrm{Zn}$ atoms with $\mathrm{Cr}$ in the layers on both the upper half and the lower half of the slab to preserve symmetry. These configurations are given in Table I, where we have identified the $\mathrm{Zn}$ sites being replaced by $\mathrm{Cr}$. In each case, we calculated the total energy corresponding to both FM and AFM spin alignment. In the latter case, the $\mathrm{Cr}$ atoms in nearest neighbors are
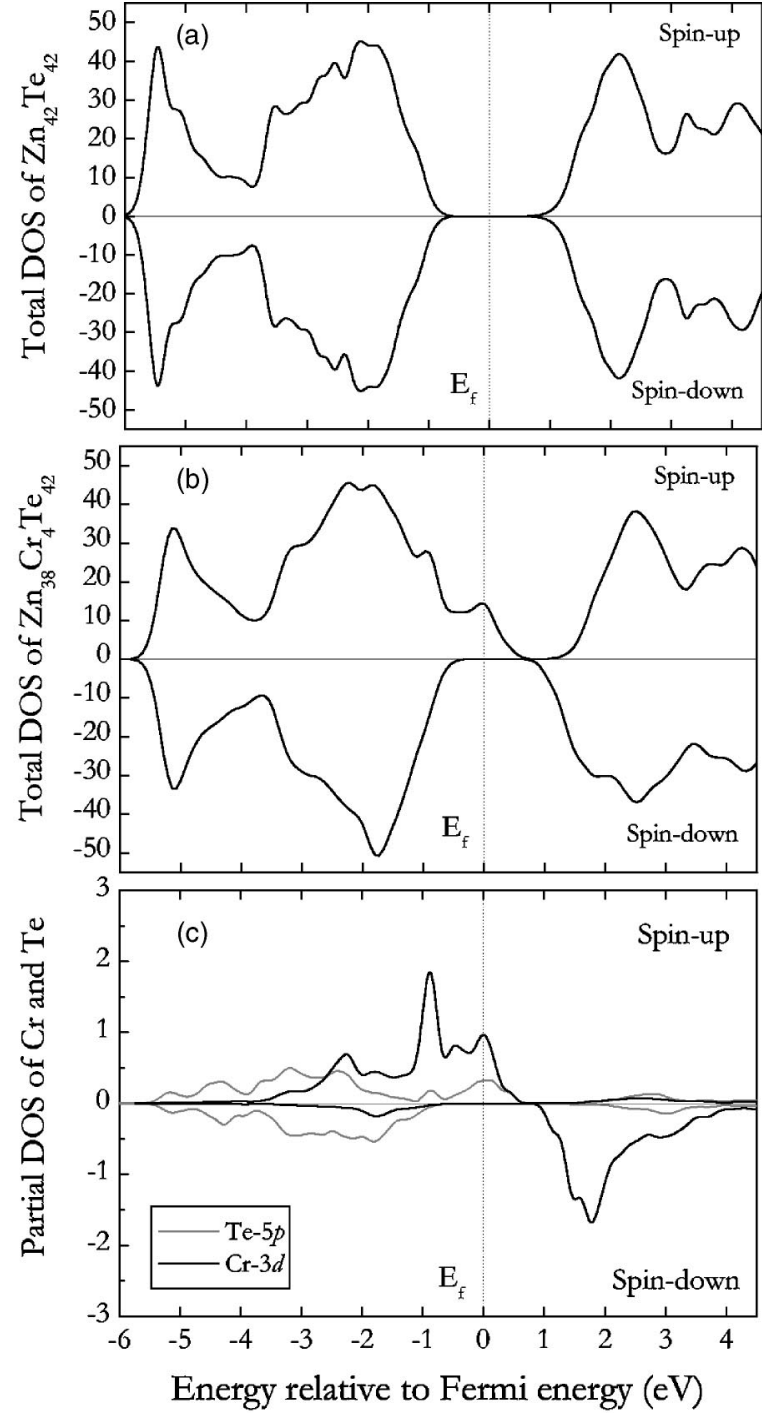

FIG. 4. (a) Total DOS of undoped ZnTe slab (corresponding to $\mathrm{Ze}_{42} \mathrm{Te}_{42}$ supercell). (b) Total DOS of $\mathrm{Zn}_{38} \mathrm{Cr}_{4} \mathrm{Te}_{42}$ slab. (c) Partial DOS of Cr $3 d$ and Te $5 p$ at neighboring sites in $\mathrm{Zn}_{38} \mathrm{Cr}_{4} \mathrm{Te}_{42}$.

assigned antiparallel spins with $5 \mu_{\mathrm{B}}$ for the initial moment, and the spin alignment is indicated by up and down arrows in the second column of Table I. For each concentration, we list the relative energies, calculated with respect to the ground state energy, for various configurations of $\mathrm{Cr}$ in Table I. The ground state energy is listed as $0.00 \mathrm{eV}$. The distance between two nearest $\mathrm{Cr}$ atoms and the magnetic moments at $\mathrm{Cr}$ and nearest Te sites corresponding to the FM phase are also given in Table I.

For the lowest energy configuration, the $\mathrm{Cr}$ atoms prefer the subsurface sites [marked Nos. $(10,12)$ in the upper half and $(33,35)$ in the lower half of the slab in Fig. 1] and form nearest neighbors. We found the FM state to be more stable than the AFM state by $0.10 \mathrm{eV} / \mathrm{Cr}$ atom. $\mathrm{Cr}$ atom carries a magnetic moment of $3.74 \mu_{\mathrm{B}}$ in the FM state. The neighboring Te atom is antiferromagnetically coupled to the $\mathrm{Cr}$ spin but carries only a very small magnetic moment, namely, $0.07 \mu_{\mathrm{B}}$. This is in agreement with the bulk system. The total DOS for the ferromagnetic ground state is shown in Fig. 4(b). Note that the Fermi energy passes through the spin-up 
TABLE I. Relative energies of the FM and AFM states of $\mathrm{Zn}_{1-x} \mathrm{Cr}_{x} \mathrm{Te}$ thin film as a function of Cr concentration. The second column identifies the $\mathrm{Zn}$ sites in Fig. 1 which are replaced by $\mathrm{Cr}$, and the arrows specify the initial spin alignment in corresponding AFM state. The $\mathrm{Cr}-\mathrm{Cr}$ distance corresponds to the optimized distance between two nearest $\mathrm{Cr}$ atoms. The magnetic moments refer to the average values per $\mathrm{Cr}$ and the nearest $\mathrm{Te}$ atom in the FM state.

\begin{tabular}{|c|c|c|c|c|c|c|}
\hline \multirow[b]{2}{*}{$\begin{array}{c}\text { Concentration } \\
\mathrm{x}\end{array}$} & \multirow[b]{2}{*}{$\begin{array}{l}\text { Configurations } \\
\text { (Cr sites) }\end{array}$} & \multirow[b]{2}{*}{$\begin{array}{c}(\mathrm{Cr}-\mathrm{Cr}) \\
\text { distance }(\AA)\end{array}$} & \multirow{2}{*}{$\frac{\text { Relative }}{\text { FM }}$} & \multirow{2}{*}{$\frac{\text { Energies }(\mathrm{eV})}{\mathrm{AFM}}$} & \multicolumn{2}{|c|}{$\begin{array}{l}\text { Magnetic moments } \\
\qquad\left(\mu_{\mathrm{B}}\right)\end{array}$} \\
\hline & & & & & $\mathrm{Cr}$ & $\mathrm{Te}$ \\
\hline \multirow[t]{5}{*}{0.095} & $\begin{aligned} \text { No. } 1 & (10,12 ; 33,35) \\
& (\uparrow \downarrow \uparrow \downarrow)\end{aligned}$ & 4.232 & 0.00 & 0.33 & 3.74 & -0.07 \\
\hline & $\begin{aligned} \text { No. } 2 & (7,10 ; 32,33) \\
& (\uparrow \downarrow \uparrow \downarrow)\end{aligned}$ & 6.101 & 0.34 & 0.39 & 3.74 & -0.04 \\
\hline & $\begin{aligned} \text { No. } 3 & (3,10 ; 38,33) \\
& (\uparrow \downarrow \uparrow \downarrow)\end{aligned}$ & 3.708 & 0.23 & 0.45 & 3.80 & -0.14 \\
\hline & $\begin{aligned} \text { No. } 4 & (2,3 ; 37,38) \\
& (\uparrow \downarrow \uparrow \downarrow)\end{aligned}$ & 3.881 & 0.51 & 0.99 & 3.79 & -0.15 \\
\hline & $\begin{aligned} \text { No. } 5 & (3,6 ; 38,40) \\
& (\uparrow \downarrow \uparrow \downarrow)\end{aligned}$ & 6.100 & 1.24 & 1.27 & 3.86 & -0.07 \\
\hline \multirow[t]{3}{*}{0.143} & $\begin{aligned} \text { No. } 1 & (11,7,8 ; 36,32,31) \\
& (\uparrow \downarrow \uparrow \uparrow \downarrow \uparrow)\end{aligned}$ & 4.316 & 0.00 & 0.36 & 3.60 & -0.08 \\
\hline & $\begin{aligned} \text { No. } 2 & (3,7,8 ; 38,32,31) \\
& (\uparrow \downarrow \uparrow \uparrow \downarrow \uparrow)\end{aligned}$ & 4.230 & 0.12 & 0.66 & 3.60 & -0.12 \\
\hline & $\begin{aligned} \text { No. } 3 & (2,3,10 ; 37,38,33) \\
& (\uparrow \downarrow \uparrow \uparrow \downarrow \uparrow)\end{aligned}$ & 3.166 & 0.21 & 0.63 & 3.75 & -0.15 \\
\hline \multirow[t]{2}{*}{0.190} & $\begin{array}{c}\text { No. } 1(2,3,10,12 ; 37,38,33,35) \\
(\uparrow \downarrow \downarrow \uparrow \uparrow \downarrow \downarrow \uparrow)\end{array}$ & 3.764 & 0.00 & 0.20 & 3.79 & -0.14 \\
\hline & $\begin{array}{c}\text { No. } 2(11,7,9,10 ; 36,32,34,33) \\
(\uparrow \downarrow \downarrow \uparrow \uparrow \downarrow \downarrow \uparrow)\end{array}$ & 4.223 & 0.19 & 1.00 & 3.74 & -0.06 \\
\hline
\end{tabular}

DOS and lies in the gap of the spin-down DOS as was seen Fig. 3. The system, therefore, is half metallic as on the bulk.

The relative energies of the FM and AFM states of $\mathrm{Zn}_{38} \mathrm{Cr}_{4} \mathrm{Te}_{42}$ supercell as well as the distance between the two nearest $\mathrm{Cr}$ atoms for the remaining four configurations are also given in Table I. Note that all these configurations lie higher in energy suggesting that $\mathrm{Cr}$ atoms prefer to cluster around a Te atom on the subsurface plane. In particular, the FM state is always lower in energy, although the difference between the FM and AFM state energies becomes smaller as the $\mathrm{Cr}-\mathrm{Cr}$ distance increases.

To understand the mechanism of FM coupling between the two $\mathrm{Cr}$ atoms doped in $\mathrm{ZnTe}$, we recall the fact that $\mathrm{Cr}_{2}$ dimer is a well-known example of AFM coupling. However, the coupling becomes FM when binding with $\mathrm{O}$ (Ref. 38) or $\mathrm{N},{ }^{39}$ and the FM coupling between $\mathrm{Cr}$ atoms in $\mathrm{Cr}_{2} \mathrm{O}$ is driven by the orbital hybridization between $\mathrm{Cr} 3 d$ and O $2 p \cdot{ }^{38} \mathrm{~A}$ similar thing also happens in $\mathrm{ZnCrTe}$. The partial densities of states at $\mathrm{Cr}$ and nearest $\mathrm{Te}$ atoms are shown in Fig. 4(c). We see that there is hybridization between $\mathrm{Cr} 3 d$ and Te $5 p$. It has been established that a critical distinguishing characteristic of a DMS is the $s p$ - $d$ exchange interaction. ${ }^{40,41}$ Therefore, confirmation of the $s p$ - $d$ exchange interaction is essential in judging whether or not the synthesized material is a DMS. The $s p$ - $d$ interaction can be investigated by magneto-optical studies such as MCD spectroscopy because magneto-optical effects are directly related to the Zeeman splitting of the band structure caused by the $s p$ - $d$ exchange interaction. ${ }^{24,40,41}$ Such $s p$ - $d$ interaction has been suggested in $\mathrm{ZnCrTe}{ }^{24-26}$ with MCD spectroscopy, which is further confirmed by our theoretical calculation. Therefore, the FM coupling between $\mathrm{Cr}$ spins in $\mathrm{ZnTe}$ results from the $\mathrm{Cr} 3 d$ and Te $5 p$ interaction.

\section{2. $\mathrm{Zn}_{0.857} \mathrm{Cr}_{0.143} \mathrm{Te}$}

This composition was simulated by replacing six $\mathrm{Zn}$ atoms with $\mathrm{Cr}$ atoms that correspond to a $\mathrm{Zn}_{36} \mathrm{Cr}_{6} \mathrm{Te}_{42}$ supercell. We have considered three configurations as listed in Table I. Once again we replaced three $\mathrm{Zn}$ sites with $\mathrm{Cr}$ in the upper half of the slab and equivalent $\mathrm{Zn}$ atoms in the lower half of the slab to preserve symmetry. The lowest energy configuration is $\mathrm{FM}$ with the three $\mathrm{Cr}$ atoms lying on the subsurface layer and forming nearest neighbors (marked Nos. 11, 7, 8 and 36, 32, 31). Each $\mathrm{Cr}$ atom has a magnetic moment of $3.60 \mu_{\mathrm{B}}$. The nearest Te atom is coupled antiferromagnetically to $\mathrm{Cr}$, but once again Te carries a very small moment. The AFM state lies $0.36 \mathrm{eV}$ higher in energy than the FM state. In the second configuration, two $\mathrm{Cr}$ atoms are in the subsurface layer, while the other occupies a surface site. In the third configuration, two $\mathrm{Cr}$ atoms are on the surface, and one $\mathrm{Cr}$ atom is in the subsurface site. For both these configurations, the FM state is again lower in energy than the AFM state. The magnetic moments per $\mathrm{Cr}$ atom are relatively unchanged and Te is weakly antiferromagnetically polarized. The total DOS as well as partial DOS at $\mathrm{Cr}$ and $\mathrm{Te}$ 
sites exhibit similar features, as shown Fig. 4, i.e. the system is half metallic, with $\mathrm{Cr} 3 d$ and $\mathrm{Te} 5 p$ contributing to the DOS at the Fermi energy.

\section{3. $\mathrm{Zn}_{0.81} \mathrm{Cr}_{0.19} \mathrm{Te}$}

In a recent experiments, ${ }^{24} \mathrm{Cr}$-doped $\mathrm{ZnTe}$ film with $\mathrm{Cr}$ concentration of $20 \%$ has been synthesized. This composition approximately corresponds to replacing eight $\mathrm{Zn}$ atoms with $\mathrm{Cr}$ atoms. We therefore considered the $\mathrm{Zn}_{34} \mathrm{Cr}_{8} \mathrm{Te}_{42}$ supercell and two different configurations for $\mathrm{Cr}$ substitution. In the first configuration, we have replaced two $\mathrm{Zn}$ sites on the first layer and two $\mathrm{Zn}$ sites on the subsurface layer by $\mathrm{Cr}$ atoms (see Table I). For the second configuration, we have replaced four nearest $\mathrm{Zn}$ atoms by $\mathrm{Cr}$ on the subsurface layer of the slab. Corresponding $\mathrm{Zn}$ atoms at the bottom half of the slab were replaced by $\mathrm{Cr}$ to preserve symmetry. The first configuration is the most stable one, in which the FM state lies $0.20 \mathrm{eV}$ lower in energy than the AFM state. In the FM state, due to lower coordination, the $\mathrm{Cr}$ atom on the surface layer carries a magnetic moment of $3.91 \mu_{\mathrm{B}}$, while on the subsurface layer, it carries a moment of $3.67 \mu_{\mathrm{B}}$. The average magnetic moment per $\mathrm{Cr}$ atom is $3.79 \mu_{\mathrm{B}}$. Te atoms in the neighborhood of $\mathrm{Cr}$ atoms are once again weakly antiferromagnetically polarized. The total and partial DOS yield the same physical picture for the electronic structure as discussed earlier for the other two concentrations.

It should be pointed out that in a typical thin-film experiment, the thickness of the film is of the order of $100 \mathrm{~nm}$, whereas we have only considered a seven-layer slab to model the thin film. To examine what relevance this model may have to real experiments, we have compared in Figs. 5(a) and 5(b) the total DOS of the slab and the partial DOS at $\mathrm{Cr}$ in the subsurface layer with that obtained from the bulk calculations. Note that the two densities of states are qualitatively the same. The magnitudes are different as the two supercells have different numbers of electrons. This suggests that $\mathrm{Cr}$ atoms on the subsurface layers behave much the same way as in the bulk and thus our slab calculation can be relevant to experimental results on thin films. On the other hand, our calculated magnetic moments are larger than the experiment values. There are several possible reasons for this. (1) We used collinear model, and noncollinear configurations are not taken into account. (2) In our calculations, the temperature is zero, while the experiments are performed in finite temperature. (3) The possible disorder in the experimental samples is not included in our calculations. However, our results are qualitatively in agreement with the experiments, and ferromagnetism in thin film is confirmed.

\section{CONCLUSION}

In summary, the magnetic coupling among $\mathrm{Cr}$ atoms doped in $\mathrm{ZnTe}$ is studied in bulk as well as in thin film. Although the magnetic moment carried by $\mathrm{Cr}$ atom near the surface $\left(3.7 \mu_{\mathrm{B}}-3.9 \mu_{\mathrm{B}}\right)$ is a little bit smaller than the moment in the bulk $\left(4 \mu_{\mathrm{B}}\right)$ due to the bond length contraction near the surface layers, FM coupling is found to be more stable than AFM coupling in both thin film and bulk. The hybridization between $\mathrm{Cr} 3 d$ and Te $5 p$ orbitals leads to ferromagnetic

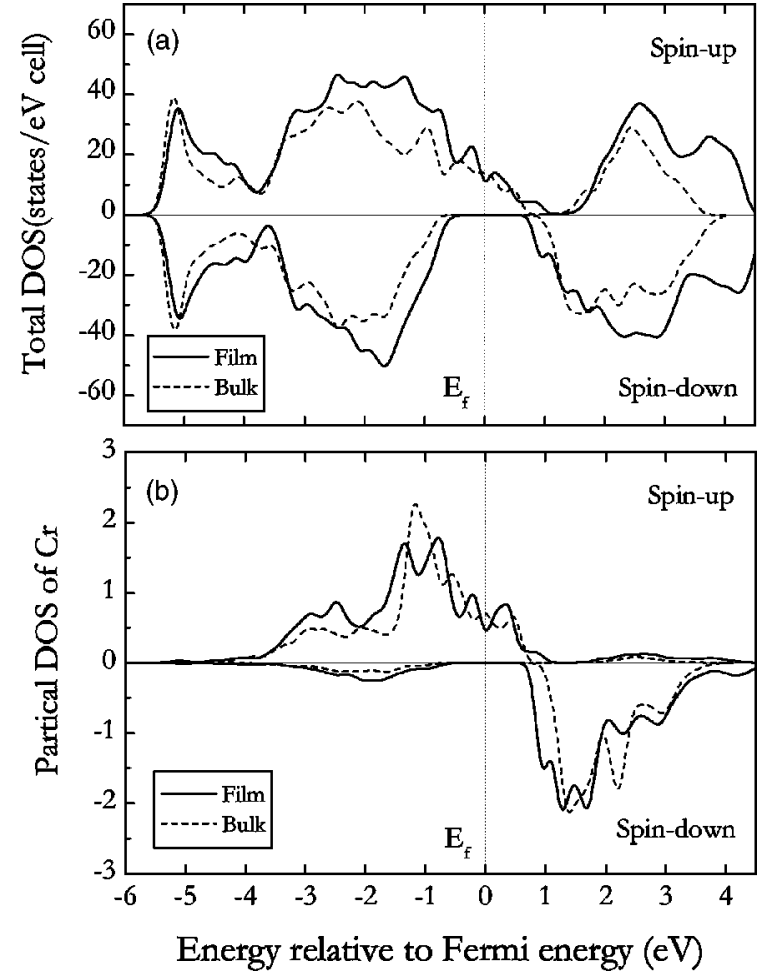

FIG. 5. (a) Total DOS of the bulk $\mathrm{Zn}_{26} \mathrm{Cr}_{6} \mathrm{Te}_{32}$ (dashed line) and the slab $\mathrm{Zn}_{34} \mathrm{Cr}_{8} \mathrm{Te}_{42}$ (solid line). (b) Partial DOS of $\mathrm{Cr} 3 d$ in the bulk $\mathrm{Zn}_{26} \mathrm{Cr}_{6} \mathrm{Te}_{32}$ (dashed line) and the slab $\mathrm{Zn}_{34} \mathrm{Cr}_{8} \mathrm{Te}_{42}$ (solid line). In both bulk and slab cases, the $\mathrm{Cr}$ concentration is closed to $20 \%$.

coupling which is similar to the cases of $\mathrm{Cr}_{2} \mathrm{O}$ and $\mathrm{Cr}_{2} \mathrm{~N}$. The $s p-d$ interaction, one of the most characteristic physical phenomena of DMS, leads to a FM coupling between the $\mathrm{Cr}$ spins in $\mathrm{Zn}_{1-x} \mathrm{Cr}_{x} \mathrm{Te}$. Our calculations on a seven-layer slab mimicking a thin film are in agreement with recent experiments.

\section{ACKNOWLEDGMENTS}

The work was supported in part by a grant from the Office of Naval Research. The authors thank the staff of the Center for Computational Materials Science, the Institute for Materials Research, Tohoku University, for their continuous support of the HITACH SR8000 supercomputing facility.

${ }^{1}$ H. Ohno, A. Shen, F. Matsukura, A. Oiwa, A. Endo, S. Katsumoto, and Y. Iye, Appl. Phys. Lett. 69, 363 (1996).

${ }^{2}$ H. Ohno, H. Munekata, S. von Molnár, and L. L. Chang, J. Appl. Phys. 69, 6103 (1991).

${ }^{3}$ T. Dietl, H. Ohno, F. Matsukura, J. Cibert, and D. Ferrant, Science 287, 1019 (2000)

${ }^{4}$ M. L. Reed, M. K. Ritums, H. H. Stadelmaier, M. J. Reed, C. A. Parker, S. M. Bedai, and N. A. El-Masry, Mater. Lett. 51, 500 (2001).

${ }^{5}$ M. L. Reed, N. A. El-Masry, H. H. Stadelmaier, M. K. Ritums, M. J. Reed, C. A. Parker, J. C. Roberts, and S. M. Bedair, Appl. Phys. Lett. 79, 3473 (2001).

${ }^{6}$ S. Sonoda, S. Shimizu, T. Sasaki, Y. Yamamoto, and H. Hori, J. Cryst. Growth 237-239, 1358 (2002).

${ }^{7}$ T. Sasaki, S. Sonoda, Y. Yamamoto, K. Suga, S. Shimizu, K. Kindo, and H. Hori, J. Appl. Phys. 91, 7911 (2002).

${ }^{8}$ G. T. Thaler, M. E. Overberg, B. Gila,R. Frazier, C. R. Abernathy, S. J. Pearton, J. S. Lee, S. Y. Lee, Y. D. Park, Z. G. Khim, J. Kim, and F. Ren, Appl. Phys. Lett. 80, 3964 (2002).

${ }^{9}$ J. M. Lee, K. I. Lee, J. Y. Chang, M. H. Ham, K. S. Huh, J. M. Myoung, W. J. Hwang, M. W. Shin, S. H. Han, H. J. Kim, and W. Y. Lee, Micro- 
electron. Eng. 69, 283 (2003).

${ }^{10}$ P. P. Chen, H. Makino, J. J. Kim, and T. Yao, J. Cryst. Growth 251, 33 (2003).

${ }^{11}$ S. S. A. Seo, M. W. Kim, Y. S. Lee, T. W. Noh, Y. D. Park, G. T. Thaler, M. E. Overberg, C. R. Abernathy, and S. J. Pearton, Appl. Phys. Lett. 82, 4749 (2003).

${ }^{12}$ Y. Shon, Y. H. Kwon, Sh. U. Yuldashev, Y. S. Park, D. J. Fu, D Y. Kim, H. S. Kim, and T. W. Kang, J. Appl. Phys. 93, 1546 (2003)

${ }^{13}$ T. Kondo, S. Kuwabara, H. Owa, and H. Munekata, J. Cryst. Growth 237-239, 1353 (2002).

${ }^{14}$ M. Hashimoto, Y. K. Zhou, M. Kanamura, and H. Asahi, Solid State Commun. 122, 37 (2002).

${ }^{15}$ S. E. Park, H. J. Lee, Y. C. Cho, S. Y. Jeong, C. R. Cho, and S. Cho, Appl. Phys. Lett. 80, 4187 (2002).

${ }^{16}$ Y. Matsumoto, M. Murakami, T. Shono, T. Hasegawa, T. Fukumura, M. Kawasaki, P. Ahmet, T. Chikyow, S. Koshihara, and H. Koinuma, Science 291, 854 (2001).

${ }^{17}$ K. Ueda, H. Tabata, and T. Kawai, Appl. Phys. Lett. 79, 988 (2001).

${ }^{18}$ G. A. Medvedkin, T. Ishibashi, T. Nishi, K. Hayata, Y. Hasegawa, and K. Sato, Jpn. J. Appl. Phys., Part 2 39, L949 (2000).

${ }^{19}$ S. W. Jung, S. J. An, G. C. Yi, C. U. Jung, S. I. Lee, and S. Cho, Appl. Phys. Lett. 80, 4561 (2002).

${ }^{20}$ P. Sharma, A. Gupta, K. V. Rao, F. J. Owens, R. Sharma, R. Ahuja, J. M. O. Guillen, B. Johansson, and G. A. Gehring, Nat. Mater. 2, 673 (2003).

${ }^{21}$ K. Ando, Appl. Phys. Lett. 82, 100 (2003).

${ }^{22}$ K. Ando, T. Hayashi, M. Tanaka, and A. Twardowski, J. Appl. Phys. 83, 6548 (1998).

${ }^{23} \mathrm{~K}$. Ando (unpublished).

${ }^{24}$ H. Saito, V. Zayets, S. Yamagata, and K. Ando, Phys. Rev. Lett. 90,
$207202(2003)$

${ }^{25}$ H. Saito, V. Zayets, S. Yamagata, and K. Ando, J. Appl. Phys. 93, 6796 (2003).

${ }^{26}$ H. Saito, V. Zayets, S. Yamagata, and K. Ando, Phys. Rev. B 66R, 081201 (2002).

${ }^{27}$ J. Blinowski, P. Kacman, and J. A. Majewski, Phys. Rev. B 53, 9524 (1996).

${ }^{28}$ K. Sato and H. Katayama-Yoshida, Semicond. Sci. Technol. 17, 367 (2002).

${ }^{29}$ E. Kulatov, Y. Uspenskii, H. Mariette, J. Cibert, D. Ferrand, H. Nakayama, and H. Ohta, J. Supercond. 16, 123 (2003).

${ }^{30}$ H. Shoren, N. Tanaka, and K. Motizuki, J. Magn. Magn. Mater. 226, 2033 (2001).

${ }^{31}$ H. Shoren, F. Ikemoto, K. Yoshida, N. Tanaka, and K. Motizuki., Physica E (Amsterdam) 10, 242 (2001).

${ }^{32}$ W. H. Xie and B. G. Liu, J. Appl. Phys. 96, 3559 (2004).

${ }^{33}$ P. E. Blőchl, Phys. Rev. B 50, 17953 (1994).

${ }^{34}$ G. Kresse and J. Heffner, Phys. Rev. B 54, 11169 (1996).

${ }^{35}$ H. J. Monkhorst and J. D. Pack, Phys. Rev. B 13, 005188 (1976).

${ }^{36}$ Q. Wang, Q. Sun, P. Jena, and Y. Kawazoe, Phys. Rev. Lett. 93, 155501 (2004).

${ }^{37}$ Q. Wang, Q. Sun, B. K. Rao, and P. Jena, Phys. Rev. B 69, 233310 (2004).

${ }^{38}$ K. Tono, A. Terasaki, T. Ohta, and T. Kondow, Phys. Rev. Lett. 90, 133402 (2003).

${ }^{39}$ Q. Wang, Q. Sun, B. K. Rao, P. Jena, and Y. Kawazoe, J. Chem. Phys. 119, 7124 (2003).

${ }^{40}$ J. K. Furdyna, J. Appl. Phys. 64, R29 (1988).

${ }^{41} \mathrm{~K}$. Ando, in Magneto-Optics, Springer Series in Solid-State Science Vol. 128, edited by S. Sugano and N. Kojima (Springer, Berlin, 2000), p. 211. 